\title{
Optimization of Sound Transmission Loss through a Thin Functionally Graded Material Cylindrical Shell
}

\begin{abstract}
Ali Nouri and Sohrab Astaraki
Department of Aerospace Engineering, Shahid Sattari Air University, Tehran 73411, Iran

Correspondence should be addressed to Ali Nouri; ali_nori@iust.ac.ir

Received 7 February 2014; Revised 10 June 2014; Accepted 22 June 2014; Published 14 July 2014

Academic Editor: Gyuhae Park

Copyright (C) 2014 A. Nouri and S. Astaraki. This is an open access article distributed under the Creative Commons Attribution License, which permits unrestricted use, distribution, and reproduction in any medium, provided the original work is properly cited.

The maximizing of sound transmission loss (TL) across a functionally graded material (FGM) cylindrical shell has been conducted using a genetic algorithm (GA). To prevent the softening effect from occurring due to optimization, the objective function is modified based on the first resonant frequency. Optimization is performed over the frequency range $1000-4000 \mathrm{~Hz}$, where the ear is the most sensitive. The weighting constants are chosen here to correspond to an A-weighting scale. Since the weight of the shell structure is an important concern in most applications, the weight of the optimized structure is constrained. Several traditional materials are used and the result shows that optimized shells with aluminum-nickel and aluminum-steel FGM are the most effective at maximizing TL at both stiffness and mass control region, while they have minimum weight.
\end{abstract}

\section{Introduction}

Functionally graded material (FGM) is a multiphase material consisting of different material components, such as ceramics and metals that have various mixture ratios and microstructures. It was first produced in Japan in the mid1980s by Yamanouchi et al. [1] and Koizumi [2]. FGM is a class of composite materials with continuous designed variation of properties throughout the volume and thus can alleviate the stress concentration found in laminated composites and can significantly enhance the thermal and mechanical properties of FGM. Furthermore, FGM can be designed to fulfill particular requirements, such as enhancing stiffness, toughness, and resistance to corrosion, wear, and high temperature by utilizing materials or material systems with various properties. Using powder metallurgy, a mixture of ceramic and metal with continuously varying volume fractions can be easily manufactured. Yamaoka et al. [3] and Zhu et al. [4] described various techniques used for the fabrication of FGMs. Consequently, in the last two decades, FGM has been developed for general use as structural components in extremely high temperature environments such as rocket engine components, military armour, space plan body, engine components, thermal barrier coating for turbine blades, and other applications [5].
Thin circular cylindrical shells are widely used in many engineering fields ranging from civil, naval, nuclear, mechanical, chemical, and aerospace applications [6]. Vibroacoustic behavior of FGM cylindrical shell is considered as an important subject for researchers. Williamson et al. [7] carried out finite element analysis and studied the residual stresses and strains in ceramic-metal joints. Moya [8] concluded that FGMs are promising candidates for future composites in aerospace, fast computers, environmental sensors, and so forth. Using a micromechanics model, Gasik and Lilius [9] calculated mechanical and thermal properties of W-Cu FGM. Sasaki and Hirai [10] carried out experiments to estimate the fatigue resistance of a Si-graphite FGM. Other researchers [11-15] carried out thermal stress analyses of FGMs under various thermal loading conditions.

A cylindrical shell structure made up of a FGM is one of the basic structural configurations where important studies have been done. Loy et al. [16, 17] carried out analysis with strain-displacement relations from Love's shell theory and the eigenvalue governing equation is obtained using the Rayleigh-Ritz method. The results demonstrated that the frequency characteristics are similar to those observed for homogeneous isotropic cylindrical shells and the frequencies are affected by the constituent volume fractions and the 
configurations of the constituent materials. Noise transmission through a composite cylindrical shell was studied by many researchers. Tang et al. [18] considered an infinite cylindrical sandwich shell excited by an oblique plane sound wave with two independent incident angles. Daneshjou et al. [19] presented an analytical solution for acoustic transmission through relatively thick FGM cylindrical shells using third order shear deformation theory.

Optimization studies of transmission loss (TL) in cylindrical shell structures with respect to their material and geometric properties have been an important topic of research for a few decades. Most of these studies have been related to composite materials. Makris et al. [20] studied transmission loss optimization in acoustic sandwich panels. The results indicated that the optimum TL is typically found at the value of the core density corresponding to the lowest of the permissible range of core densities established at the start of the optimization search. Wang et al. [21] used a genetic algorithm to optimize a sandwich panel with balanced acoustic and mechanical properties at a minimal weight. The optimization of sound transmission through FGM cylindrical shells that were subjected to plane sound wave has not been studied yet. In the present work, an attempt is made to address this problem. Therefore, it is very important to develop an accurate, reliable analysis towards the understanding of the optimization sound transmission through FGM cylindrical shells. Therefore, this paper presents a study on the optimization of sound transmission loss across FGM cylindrical shell using a genetic algorithm. A parametric study of sound transmission through a FGM shell excited by an incident oblique plane sound wave was developed and considered as objective function to determine which combinations of design variables could result in a FGM shell which maximizes the sound insulation. The result shows that an optimized shell with aluminum-nickel or aluminum-steel FG has the largest $\mathrm{TL}$ at both stiffness and mass control region while having minimum weight.

\section{Model Specification}

The specific problem is an oblique plane wave impinging on a flexible FGM thin cylindrical shell of infinite length and includes the reflection and scattering of the incident wave and the effect of an external airflow in the $x$-direction (Figure 1). The angle of incidence of the wave is $\gamma$ (measured from the axial axis of cylinder), and the incidence wave approaches from the $\theta=\pi$ direction. The inside cavity was assumed to be anechoic and only inward-traveling waves exist. In the analysis, all waves will be assumed to have the same dependence on the axial coordinate $z$ and the cylinder will be assumed infinitely long [22]. The fluid media in the external and the internal space are defined by the density and the speed of sound: $\left\{\rho_{1}, c_{1}\right\}$ and $\left\{\rho_{3}, c_{3}\right\}$, respectively.

2.1. Functionally Graded Material. In order to model FG properties for a cylindrical shell composed of $m$ different materials with a uniform thickness $h$ and a middle reference

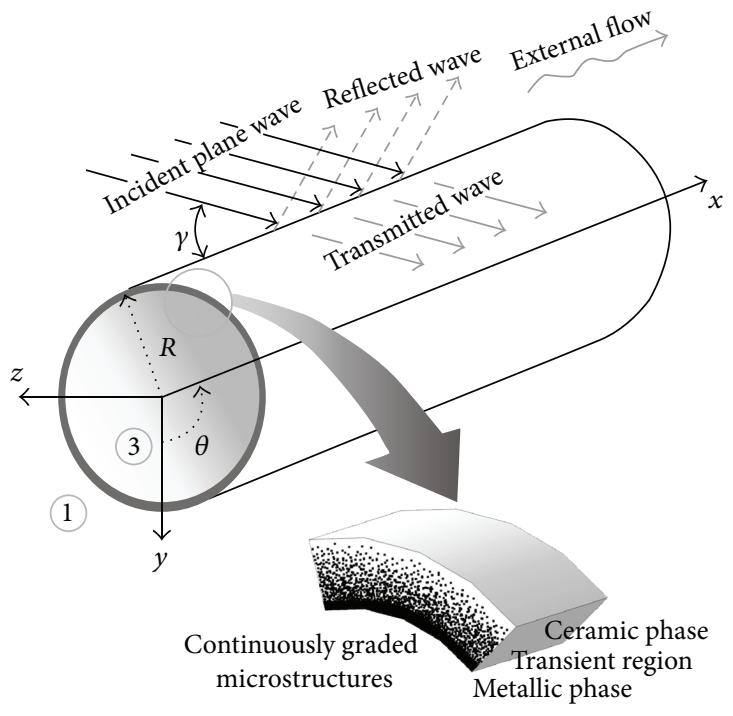

FIGURE 1: Schematic diagram of the FGM cylindrical shell.

surface, the volume fraction relation $V_{f}$ changes continuously through the thickness direction, considered as [16]

$$
\begin{gathered}
\sum_{k=1}^{m} V_{f}=1, \\
V_{f}=\left(\frac{z+h / 2}{h}\right)^{N},
\end{gathered}
$$

where $N$ is the power-law exponent $(0 \leq N \leq \infty)$. The general formulation for a FGM composed of two components can be set as [17]

$$
X=\left(X_{1}-X_{2}\right)\left(\frac{z+h / 2}{h}\right)^{N}+X_{2}
$$

where $X$ can be substituted with $E, \rho$, or $v$ in (2) to obtain the elasticity modulus, mass density, and poisson's ratio for the FGM cylindrical shell, respectively. From these equations, when $z=h / 2, E=E_{2}, \rho=\rho_{2}, \nu=\nu_{2}$, and when $z=-h / 2, E=E_{1}, \rho=\rho_{1}, v=v_{1}$. The material properties vary continuously from material 2 at the inner surface of the cylindrical shell to material 1 at the outer surface of the cylindrical shell.

2.2. Fluid Equations. Due to the existence of airflow in the external fluid medium, the external pressure, which is the summation of the incident wave $p_{1}^{I}$ and the reflected wave $p_{1}^{R}$, satisfies the following wave equation [23]:

$$
c_{1}^{2} \nabla^{2}\left(p_{1}^{I}+p_{1}^{R}\right)-\left(\frac{\partial}{\partial t}+V \cdot \nabla\right)^{2}\left(p_{1}^{I}+p_{1}^{R}\right)=0,
$$

where $\nabla^{2}$ is the Laplacian operator in the cylindrical coordinate system. The internal pressure of cavity satisfies the acoustic wave equation:

$$
C_{3}^{2} \nabla^{2} p_{3}^{T}-\frac{\partial^{2} p_{3}^{T}}{\partial t^{2}}=0
$$

where $p_{3}^{T}$ is the transmitted wave. 
As a result of considering Helmholtz equations in internal and external space and also the equality of particle velocities of the acoustic media and the shell, the boundary conditions of the model can be defined as

$$
\begin{gathered}
\left.\frac{\partial\left(p_{1}^{I}+p_{1}^{R}\right)}{\partial r}\right|_{r=R}=-\rho\left(\frac{\partial}{\partial t}+V \cdot \nabla\right)^{2} W, \\
\left.\frac{\partial p_{3}^{T}}{\partial r}\right|_{r=R}=-\rho \frac{\partial^{2} W}{\partial t^{2}} .
\end{gathered}
$$

2.3. Formulation. Consider a cylindrical shell with radius $R$ and thickness $h$; see Figure 1 . The deformations defined with reference to a coordinate system, taken at the middle surface, are $u, v$, and $w$ in the axial, circumferential, and radial directions, respectively. For a thin cylindrical shell, plane stress condition is assumed and the constitutive relation is given by

$$
\{\sigma\}=[Q]\{\varepsilon\}
$$

The stress vector, strain vector, and the reduced stiffness matrix are defined as

$$
\begin{gathered}
\{\sigma\}^{T}=\left\{\begin{array}{lll}
\sigma_{x} & \sigma_{\theta} & \sigma_{x \theta}
\end{array}\right\}, \\
\{\varepsilon\}^{T}=\left\{\begin{array}{lll}
\varepsilon_{x} & \varepsilon_{\theta} & \varepsilon_{x \theta}
\end{array}\right\}, \\
{[Q]=\left[\begin{array}{ccc}
Q_{11} & Q_{12} & 0 \\
Q_{12} & Q_{22} & 0 \\
0 & 0 & Q_{66}
\end{array}\right] .}
\end{gathered}
$$

For isotropic materials, the reduced stiffness $Q_{i j}(i, j=1,2$ and 6) is defined as

$$
\begin{aligned}
& Q_{11}=Q_{22}=\frac{E}{1-v^{2}}, \\
& Q_{12}=\frac{v E}{1-v^{2}}, \\
& Q_{66}=\frac{E}{2(1+v)} .
\end{aligned}
$$

From Love's shell theory, the components in the strain vector are defined as $[23,24]$

$$
\varepsilon_{x}=e_{1}+z \kappa_{1}, \quad \varepsilon_{\theta}=e_{2}+z \kappa_{2}, \quad \varepsilon_{x \theta}=\gamma+2 z \tau,
$$

where $e_{1}, e_{2}$, and $\gamma$ are the reference surface strains and $\kappa_{1}$, $\kappa_{2}$, and $\tau$ are the surface curvatures. These surface strains and curvatures are defined as

$$
\begin{gathered}
\left\{e_{1}, e_{2}, \gamma\right\}=\left\{\frac{\partial u}{\partial x}, \frac{1}{R}\left(\frac{\partial v}{\partial \theta}+w\right), \frac{\partial v}{\partial x}+\frac{1}{R} \frac{\partial u}{\partial \theta}\right\}, \\
\left\{\kappa_{1}, \kappa_{2}, \tau\right\}=\left\{-\frac{\partial^{2} w}{\partial x^{2}},-\frac{1}{R^{2}}\left(\frac{\partial^{2} w}{\partial \theta^{2}}-\frac{\partial v}{\partial \theta}\right),\right. \\
\left.\frac{\partial v}{\partial x}+\frac{1}{R}\left(\frac{\partial^{2} w}{\partial x \partial \theta}-\frac{\partial v}{\partial x}\right)\right\} .
\end{gathered}
$$

For a thin cylindrical shell, the force and moment resultants are defined as

$$
\begin{gathered}
\left\{N_{x}, N_{\theta}, N_{x \theta}\right\}=\int_{-h / 2}^{h / 2}\left\{\sigma_{x} \sigma_{\theta} \sigma_{x \theta}\right\} d z, \\
\left\{M_{x}, M_{\theta}, M_{x \theta}\right\}=\int_{-h / 2}^{h / 2}\left\{\begin{array}{lll}
\sigma_{x} & \sigma_{\theta} & \sigma_{x \theta}
\end{array}\right\} z d z .
\end{gathered}
$$

Substituting (7), with substitution from (10), into (12), the constitutive equation is obtained as

$$
\left\{\begin{array}{c}
N_{x} \\
N_{\theta} \\
N_{x \theta} \\
M_{x} \\
M_{\theta} \\
M_{x \theta}
\end{array}\right\}=\left[\begin{array}{cccccc}
A_{11} & A_{12} & 0 & B_{11} & B_{12} & 0 \\
A_{12} & A_{22} & 0 & B_{12} & B_{22} & 0 \\
0 & 0 & A_{66} & 0 & 0 & B_{66} \\
B_{11} & B_{12} & 0 & D_{11} & D_{12} & 0 \\
B_{12} & B_{22} & 0 & D_{12} & D_{22} & 0 \\
0 & 0 & B_{66} & 0 & 0 & D_{66}
\end{array}\right]\left\{\begin{array}{c}
e_{1} \\
e_{2} \\
\gamma \\
\kappa_{1} \\
\kappa_{2} \\
\tau
\end{array}\right\},
$$

where $A_{i j}, B_{i j}$, and $D_{i j}(i, j=1,2$ and 6$)$ are the extensional, coupling, and bending stiffness defined as

$$
\left\{A_{i j}, B_{i j}, D_{i j}\right\}=\int_{-h / 2}^{h / 2} Q_{i j}\left\{1, z, z^{2}\right\} d z .
$$

According to $[23,24]$, a thin cylindrical shell's equations of motion in cylindrical coordinate are as follows:

$$
\begin{gathered}
-\frac{N_{\theta}}{R}+\frac{\partial^{2} N_{z}}{\partial z^{2}}+\frac{2}{R} \frac{\partial^{2} M_{z \theta}}{\partial z \partial \theta} \\
+\frac{1}{R}\left[\frac{1}{R} \frac{\partial^{2} M_{\theta}}{\partial \theta^{2}}+\frac{\partial M_{z \theta}}{\partial z}\right]+q_{y}=-\bar{M} \frac{\partial^{2} w}{\partial t^{2}} \\
\frac{1}{R} \frac{\partial N_{\theta}}{\partial \theta}+\frac{\partial N_{z \theta}}{\partial z} \\
+\frac{1}{R}\left[\frac{1}{R} \frac{\partial M_{\theta}}{\partial \theta}+\frac{\partial M_{z \theta}}{\partial z}\right]+q_{\theta}=-\bar{M} \frac{\partial^{2} v}{\partial t^{2}} \\
\frac{\partial N_{z}}{\partial z}+\frac{1}{R} \frac{\partial^{2} N_{z \theta}}{\partial z \partial \theta}+q_{z}=-\bar{M} \frac{\partial^{2} u}{\partial t^{2}} \\
\bar{M}=\int_{-h / 2}^{h / 2} \rho d z,
\end{gathered}
$$

where $q_{z}, q_{\theta}$, and $q_{y}$ are loads in axial, circumferential, and radial direction, respectively.

2.4. Vibroacoustic Solution. The harmonic plane wave $p^{I}$ in cylindrical coordinate incident from outside of the shell to the direction shown in Figure 1 can be expressed as [25]

$$
\begin{aligned}
p^{I} & (r, z, \theta, t) \\
& =P_{0} \sum_{n=0}^{\infty} \varepsilon_{n}(-j)^{n} J_{n}\left(k_{1 r} r\right) \cos (n \theta) \exp \left[j\left(\omega t-k_{1 z} z\right)\right],
\end{aligned}
$$

where $k_{1}$ is the wave number in the external medium of shell and $J_{n}$ is the Bessel function of the first kind of integer order 
$n, \varepsilon_{n}$ is the Neumann factor, $P_{0}$ is the amplitude of the incident wave, $j=-1, n=0,1,2,3, \ldots$, and $\omega$ is the angular frequency. The waves radiated from the shell to the outside and into the cavity, $p_{1}^{R}$ and $p_{3}^{T}$, can be represented as

$$
\begin{aligned}
p_{1}^{R}(r, z, \theta, t) & \\
= & \sum_{n=0}^{\infty} P_{1 n}^{R} H_{n}^{2}\left(k_{1 r} r\right) \exp \left[j\left(\omega t-k_{1 z} z-n \theta\right)\right], \\
p_{3}^{T}(r, z, \theta, t) & \\
= & \sum_{n=0}^{\infty} P_{3 n}^{T} H_{n}^{1}\left(k_{3 r} r\right) \exp \left[j\left(\omega t-k_{3 z} z-n \theta\right)\right],
\end{aligned}
$$

where $H_{n}^{2}, H_{n}^{1}$ are the Hankel functions of the first and second kind of integer order $n$, respectively. Three components of the shell displacements can be expressed as [24]

$$
\begin{aligned}
& w(z, \theta, t)=\sum_{n=0}^{\infty} W_{n} \exp \left[j\left(\omega t-k_{1 z} z-n \theta\right)\right], \\
& u(z, \theta, t)=j \sum_{n=0}^{\infty} U_{n} \exp \left[j\left(\omega t-k_{1 z} z-n \theta\right)\right], \\
& v(z, \theta, t)=j \sum_{n=0}^{\infty} V_{n} \exp \left[j\left(\omega t-k_{1 z} z-n \theta\right)\right] .
\end{aligned}
$$

Because the traveling waves in the acoustic media and inside the shell are driven by the incident traveling wave, the wave numbers (or trace velocities) in the $z$ direction should match throughout the system; therefore, $k_{3 z}=k_{1 z}$.

Equations (16)-(18) are substituted into the three equations of motion and two boundary conditions ((5)-(6)). These five equations involve six variables: the amplitudes of the outgoing and incoming waves in the exterior cavity, the transmitted wave in the interior cavity, and three displacements of the shell structure. Therefore, the solutions can be obtained as the ratios to the one of the variables, in this case, the pressure amplitude of the incoming wave [18].

2.5. Sound Transmission Loss. Sound transmission loss (STL) can be defined as the ratio of the incoming and transmitted sound powers per unit length of the cylinder. Consider

$$
\mathrm{STL}=10 \log _{10} \frac{W^{I}}{W^{T}},
$$

where $W^{T}$ and $W^{I}$ are the transmitted and incident power flow per unit length of the shell. The equations for $W^{T}$ and $W^{I}$ are developed by Kim and Lee [25]. However, because of the incident angle in the present studies, the equation of Kim and Lee's work should be changed to

$$
W^{I}=\frac{\cos (\gamma) p_{0}^{2}}{\rho_{1} c_{1}} R
$$

Therefore, the final quantity of transmission loss can be defined as

$$
\mathrm{STL}=-10 \log _{10} \tau_{\mathrm{si}}
$$

where

$$
\tau_{\mathrm{si}}=\sum_{n=0}^{\infty} \frac{\operatorname{Re}\left\{p_{3 n}^{T} \times H_{n}^{1}\left(k_{3 r} R\right)\left(j \omega W_{n}^{0}\right)^{*}\right\} \times \rho_{1} c_{1} \pi}{\varepsilon_{n} \cos \gamma p_{0}^{2}} .
$$

\section{The Optimization Problem}

To optimally design a structure so that its STL must be minimal, we have to deal with a multivariable constrained nonlinear optimization problem. The optimization problem can be stated as

$$
\begin{array}{ll}
\min & f(X)=-\operatorname{STL}(X), \quad X=\left[x_{1}, x_{2}, \ldots, x_{n}\right], \quad X \in R^{n}, \\
\text { s.t. } & g_{i}(X)=0, \quad i=1, \ldots, m, \\
& c_{i}(X) \leq 0, \quad i=m+1, \ldots, p,
\end{array}
$$

where the $g_{i}$ are $m$ equality constraints and the $c_{i}$ are $(p$ $m$ ) inequality constraints. $f(x)$ is the objective function to be minimized with respect to the vector $x$ of the design variables. In the present paper, we study the optimization problem of the shell with respect to the geometric and material properties. The design variables for this problem are chosen as

$$
X=[R, h, N] X_{\mathrm{lb}} \leq X \leq X_{\mathrm{ub}},
$$

where $R, h$, and $N$ are radius, thickness (geometric design variable), and power-law exponent, respectively. $X_{\mathrm{lb}}$ and $X_{\mathrm{ub}}$ are vectors which define the lower and upper bounds of the design variables. Since the weight of the shell structure is an important concern in most applications, a constraint is introduced as follows:

$$
\begin{aligned}
& c_{1}=\int_{-h / 2}^{h / 2}\left(\left(\rho_{1}-\rho_{2}\right)\left(\frac{2 z+h}{h}\right)^{N}+\rho_{2}\right) d z-m_{0} \leq 0, \\
& c_{2}=\frac{h}{R}-0.05 \leq 0,
\end{aligned}
$$

where $m_{0}$ is a constant and refers to the mass per unit length of shell.

A large number of optimization techniques exist for different problems. In this study, the genetic algorithm is used as optimization method. The genetic algorithm is a method for solving both constrained and unconstrained optimization problems that is based on natural selection, the process that drives biological evolution. It repeatedly modifies a population of individual solutions. At each step, the genetic algorithm selects individuals at random from the current population to be parents and uses them to produce the children for the next generation. Over successive generations, the population "evolves" toward an optimal solution. The genetic algorithm uses three main types of rules at each step to create the next generation from the current population: selection rules select the individuals, called parents that contribute to the population at the next generation. Crossover rules combine two parents to form children for the next generation. Mutation rules apply random changes to individual parents to form children. 
TABLE 1: Material and environmental properties.

\begin{tabular}{|c|c|c|c|c|c|c|c|}
\hline \multirow{2}{*}{ Material } & \multicolumn{5}{|c|}{ Shell materials } & \multirow{2}{*}{$\begin{array}{c}\text { Cavity } \\
\text { Air }\end{array}$} & \multirow{2}{*}{$\begin{array}{c}\text { Ambient } \\
\text { Air }\end{array}$} \\
\hline & Aluminum & Steel & Nickel & Zirconia & Brass & & \\
\hline$\rho\left(\mathrm{kg} / \mathrm{m}^{3}\right)$ & 2760 & 7750 & 8900 & 5700 & 8500 & 2.9 & 2.9 \\
\hline$E(\mathrm{Gpa})$ & 72 & 210 & 224 & 244 & 104 & - & - \\
\hline$v$ & 0.33 & 0.3 & 0.34 & 0.3 & 0.37 & - & - \\
\hline Sound speed (m/s) & - & - & - & - & - & 340 & 340 \\
\hline Incident angle (deg.) & & & & 45 & & & \\
\hline Shell radius $(\mathrm{m})$ & & & & 1.5 & & & \\
\hline Shell thickness (mm) & & & & 1.5 & & & \\
\hline$m_{0}(\mathrm{~kg} / \mathrm{m})$ & & & & $6.21\left(1.5 \times m_{\mathrm{Al}}\right)$ & & & \\
\hline
\end{tabular}

\section{Result and Discussion}

Numerical simulations have been carried out to study the optimization problem of a FGM cylindrical shell. With proper qualitative interpretations, the theoretical model developed can be used very effectively in the basic design stage of cylinder-shape vibroacoustic systems. As a demonstration of such applications, design parameter studies are conducted. The basic shell dimensions and simulation conditions used in the study are listed in Table 1.

The TL of a FGM Brass-Al shell is illustrated in Figure 2. As shown, three important frequencies are recognized: ring frequency $f_{r}$ (where the wavelength of a longitudinal wave in the shell is equal to the circumference), critical pseudocoincidence $f_{\mathrm{pc}}$ (spatial coincidence in radial direction between the wave vector projection of excitation and the shell circumferential wave number), and coincidence frequency $f_{c}$ (where the trace velocity of the acoustic wave is equal to the bending wave velocity in the shell wall). The various zones controlled by the stiffness $\left(0-f_{r}\right)$, the mass $\left(f_{r}-f_{\mathrm{pc}}\right)$, and the coincidence $\left(f>f_{c}\right)$ and the resonant modes zone enclosed between $f_{\mathrm{pc}}$ and $f_{c}$ will be noted [26].

Figure 3 shows the effect of the shell materials on the STL. Materials chosen for the comparison are aluminum, steel, nickel, zirconia, and brass as shown in Table 1. The figure shows that the brass is the most effective in the high frequency range and zirconia is the most effective in the low frequency range. This is as expected because the density of the brass and the stiffness of the zirconia are the largest, which make them most effective in the mass and stiffness controlled regions, respectively. The figure also shows that the aluminum, which has the lowest stiffness, is the least effective in the low frequency range, which is again as expected because the low frequency range is controlled by the stiffness. Steel and nickel have the same behavior in the low and high frequency range and are more effective in both mass and stiffness controlled regions.

Although steel and nickel are more effective, the structural weight of a cylindrical shell that is made of them is too high. Since the weight of the shell structure is an important concern in most applications, it is necessary to compromise between sound transmission loss of cylindrical shell and its weight. The results, however, lead to a structure with a lower stiffness than the base shell. To prevent the softening

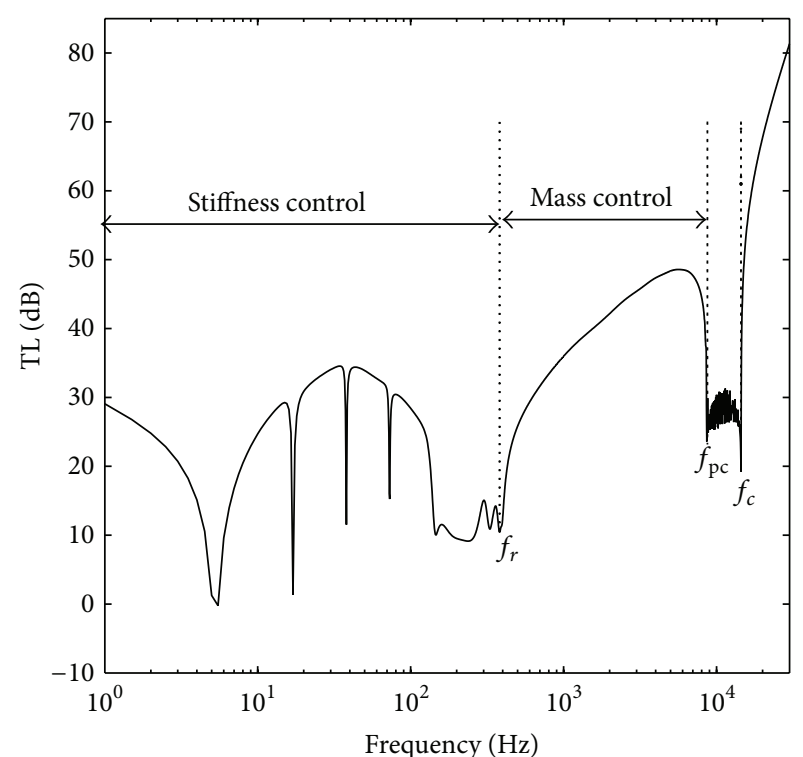

Figure 2: The TL of FGM shell.

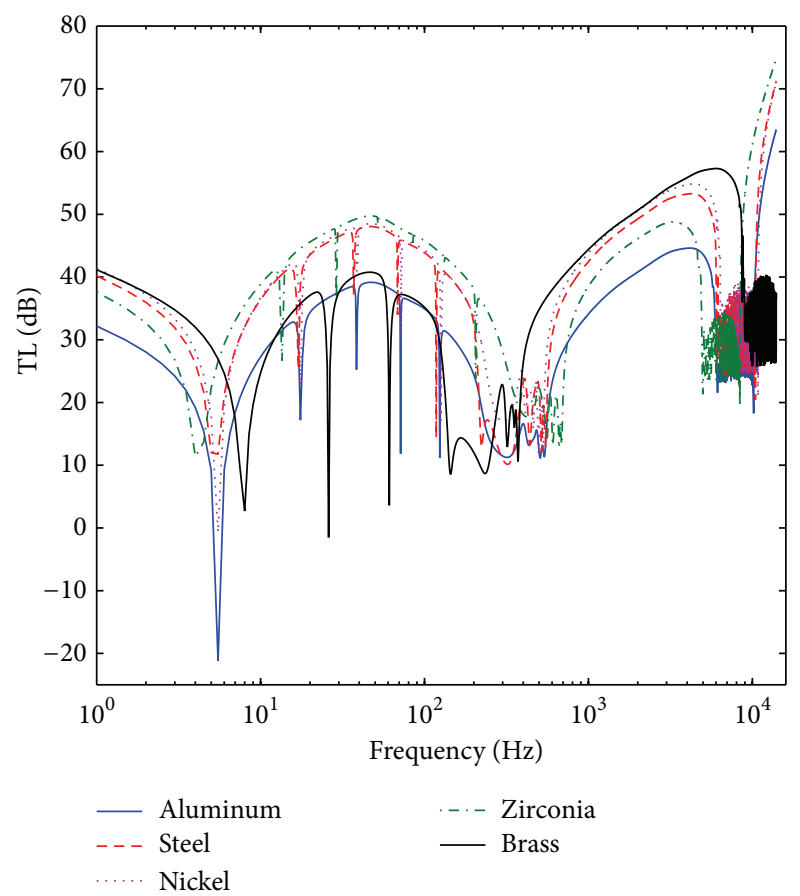

FIGURE 3: Effect of different materials on STL. 
TABLE 2: Effect of $\beta$ values on the design parameters of the optimized shell.

\begin{tabular}{lccccc}
\hline$\beta$ & $R(\mathrm{~m})$ & $h(\mathrm{~mm})$ & $N$ & $f_{1}(\mathrm{~Hz})$ & STL $(\mathrm{db})$ \\
\hline 0 & 1.6 & 0.0013 & 0.393 & 4 & 38.6 \\
0.4 & 1.58 & 0.00143 & 0.647 & 5.5 & 38.42 \\
0.5 & 1.51 & 0.00151 & 0.817 & 6 & 38.57 \\
0.6 & 1.45 & 0.00167 & 1.3 & 6 & 38.44 \\
\hline
\end{tabular}

effect from occurring due to optimization, we introduce an additional term in the objective function:

$$
\min f(X)=(1-\beta)(-\operatorname{STL}(X))+\beta\left(f o_{1}-f(X)\right),
$$

where $f(x)$ is the first resonant frequency of the optimized shell, $f o_{1}=7 \mathrm{~Hz}$ is the first resonant frequency of the base shell, and $\beta$ is a weighting constant. Note that the first resonant frequency of a structure is a common measure of the stiffness of the structure. This modified objective function will penalize the reduction of the stiffness of the structure. The optimization is then performed at octave band frequency under $1000 \mathrm{~Hz}$. The results evaluate an equal weighting of $\beta=0,0.4,0.5,0.6$ given to both the sound transmission loss and the stiffness of the structure. The optimization results for other values of $\beta$ are similar and are not presented. Table 2 shows the results of the optimized shell at the frequency. In this example, stainless steel and aluminum are considered as the constituent materials of the FGM cylindrical shell.

The results indicate that the optimum values are typically found at the value of $\beta=0.5$, so it is used for other analyses in this study. The results shown in Table 2 are the shell optimized at discrete frequency. It is also common to optimize a frequency-weighted average of the transmission loss. For practical purposes hearing encompasses a range of frequencies from about $16 \mathrm{~Hz}$ to somewhat less than $20000 \mathrm{~Hz}$. However, low frequencies do not affect humans very strongly, while those from $500-5000 \mathrm{~Hz}$ (where the ear is most sensitive) are very important.

For the sound transmission class (STC) - the singlenumber rating system which compares the sound TL of a test specimen with a standard contour-the following set of 16 frequencies is used: $125,160,200,250,315,400,500,630,800$, $1000,1250,1600,2000,2500,3150$, and $4000 \mathrm{~Hz}$ [27].

This study uses the frequency range $1-4 \mathrm{KHz}$. However, instead of the 21 frequencies $(150 \mathrm{~Hz}$ steps) used then, only the seven standardized frequencies from the STC rating in the 1000 to $4000 \mathrm{~Hz}$ range will be incorporated. Namely, the following frequencies are considered: 1000, 1250, 1600, 2000, 2500,3150 , and $4000 \mathrm{~Hz}$. The smaller number of frequencies does not lessen the accuracy in the calculations of TL because a sophisticated integration algorithm was used to find the field-incidence average TL. Also, the use of these frequencies parallels the portion of the spectrum emphasized in the recent ASTM standard on isolation between neighboring rooms [25]. The lower limit of the range $(1000 \mathrm{~Hz})$ was chosen
TABLE 3: Normalized weights using the A-weighting.

\begin{tabular}{lccc}
\hline Frequency $(\mathrm{Hz})$ A-weighting $\mathrm{dB}(\mathrm{A})$ & Multiplier $\mathbf{1 0} \mathbf{\mathbf { d B } ( \mathbf { A } ) / \mathbf { 1 0 }}$ & $\beta_{i}$ \\
\hline 1000 & 0 & 1 & 0.1156 \\
1250 & 0.6 & 1.148 & 0.1327 \\
1600 & 1 & 1.259 & 0.1455 \\
2000 & 1.2 & 1.318 & 0.1524 \\
2500 & 1.3 & 1.349 & 0.1559 \\
3150 & 1.2 & 1.318 & 0.1524 \\
4000 & 1 & 1.259 & 0.1455 \\
\hline
\end{tabular}

TABLE 4: FGM combinations.

\begin{tabular}{lcc}
\hline FGM & Inner surface & Outer surface \\
\hline Case 1 & Aluminum & Steel \\
Case 2 & Aluminum & Nickel \\
Case 3 & Aluminum & Zirconia \\
Case 4 & Aluminum & Brass \\
\hline
\end{tabular}

in part because of experimental results cited by Dym and Lang [28]. In this case, the objective function is modified as

$$
\begin{gathered}
\min f(X)=(1-\beta)\left(-\mathrm{STL}_{\mathrm{avg}}(X)\right)+\beta\left(f o_{1}-f(X)\right), \\
\operatorname{STL}_{\mathrm{avg}}(X)=-10 \log _{10}\left|\tau_{\mathrm{avg}}(X)\right|, \\
\tau_{\mathrm{avg}}(X)=\sum_{i=0}^{N_{f}} \bar{\beta}_{i} \tau_{\mathrm{si}}(X),
\end{gathered}
$$

where $\bar{\beta}_{i}$ represent weighting constants and $N_{f}$ is the number of frequencies [29]. $\bar{\beta}_{i}$ are normalized so that the sum of all the coefficients is unity. The weighting constants $\bar{\beta}_{i}$ are commonly chosen to correspond to an A-weighting. It should be mentioned here that with A-weighting, the sound-level meter is relatively less sensitive to sounds of frequency below 1000 and above $4000 \mathrm{~Hz}$. The weighting and discrete frequencies that were chosen in the bound $1-4 \mathrm{kHz}$ are identical to those used to defined single insulation measure in an ASTM standard $[27,28]$. The normalized weights $\bar{\beta}_{i}$ are given in Table 3.

In this study, based on the materials listed in Table 1, different functionally graded cylindrical shells (Table 4) should be optimized based on (27) and (24)-(25).

As the weight of structure is an important parameter in aerospace vehicles, aluminum is used as inner surface of constituent materials because its density is lowest among the metals in Table 1. The optimized results of the all cases are tabulated in Table 5.

The result shows that case 2 is the most effective for the STL and case 3 is worse than the other cases. Figure 4 shows the variation of the STL for the all cases with optimized design parameters of Table 5.

As indicated, the results are different for the optimized shells with different kinds of FGM, although STL improvement is achieved in all cases. As obviously illustrated in Figure 4, TLs characteristics have been significantly improved 


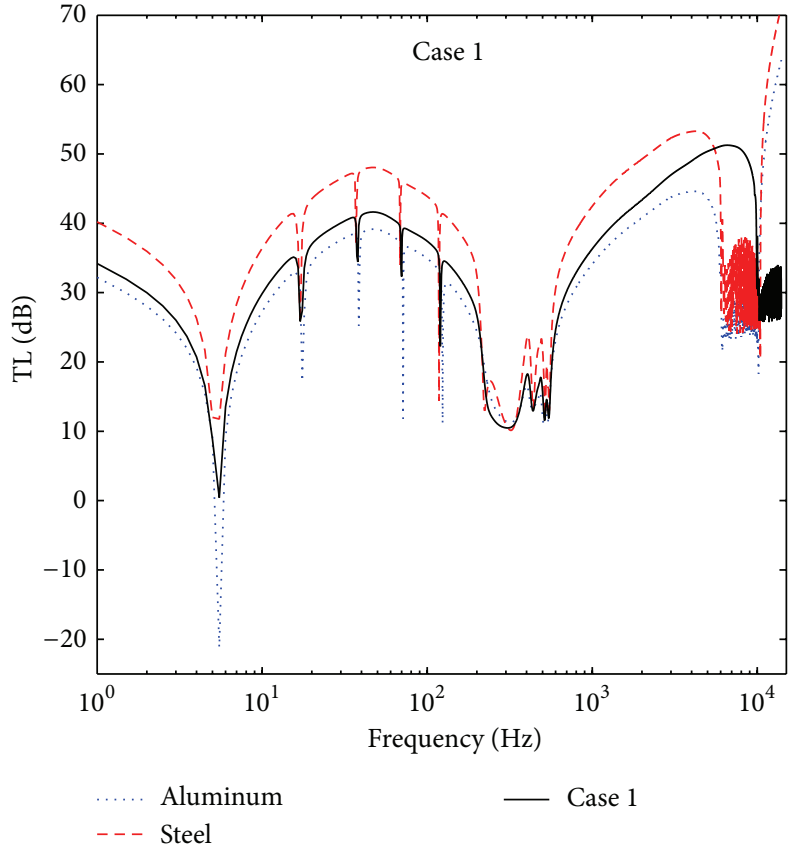

(a)

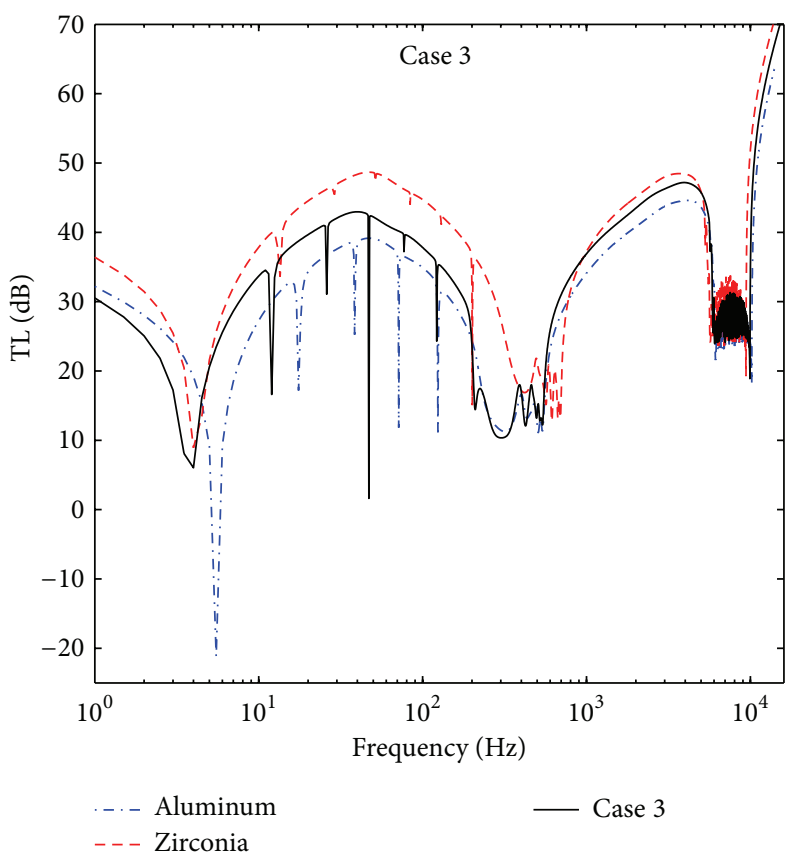

(c)

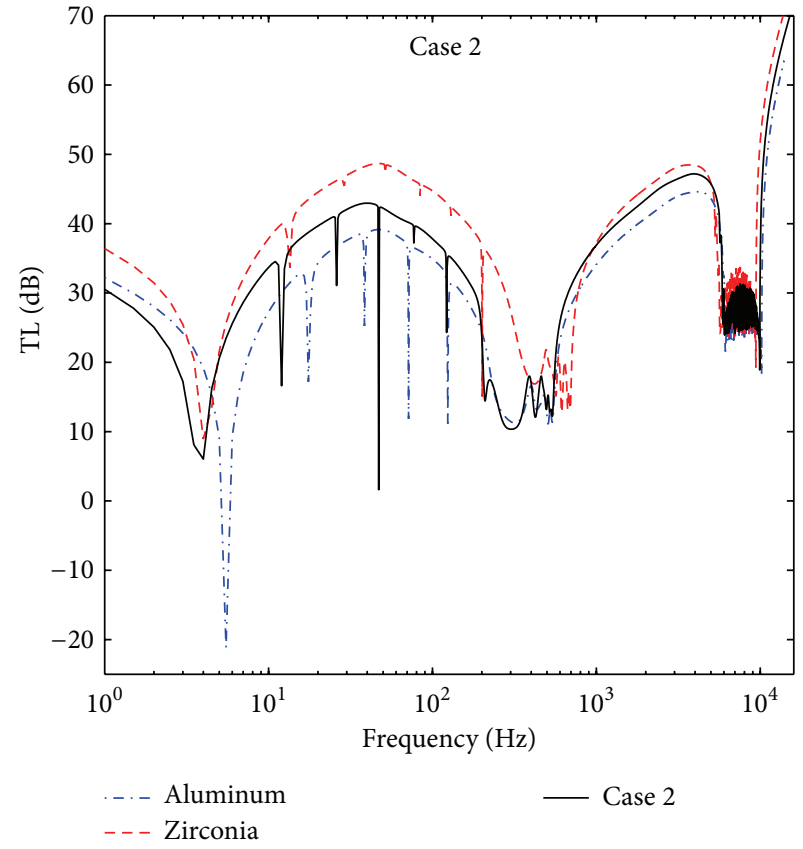

(b)

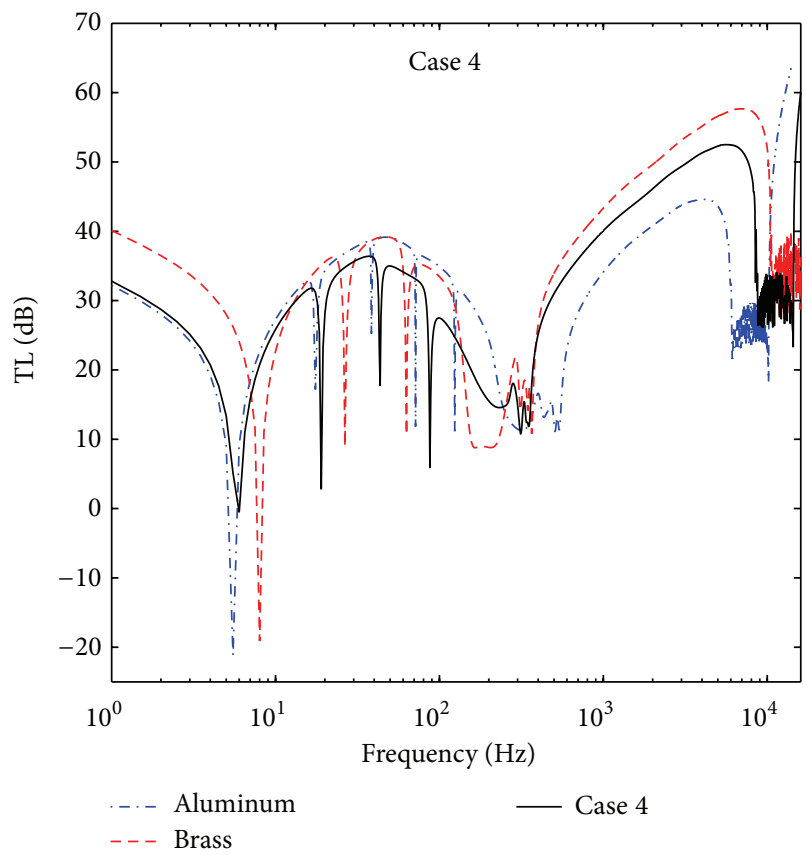

(d)

FIgURE 4: TL as a function of frequency $f$ for the FGM shells listed in Table 4.

TABLE 5: Optimized value of FGM combinations.

\begin{tabular}{lccccc}
\hline Condition & $h(\mathrm{~mm})$ & $N$ & $f_{1}(\mathrm{~Hz})$ & $R(\mathrm{~m})$ & $\sum$ STL at frequencies of Table 3 \\
\hline Case 1 & 13 & 1.59 & 5.5 & 1.6 & 301.91 \\
Case 2 & 11 & 1.37 & 6 & 1.5 & 312.89 \\
Case 3 & 16 & 0.84 & 4 & 1.8 & 298.34 \\
Case 4 & 14 & 1.2 & 5 & 1.8 & 321.73 \\
\hline
\end{tabular}




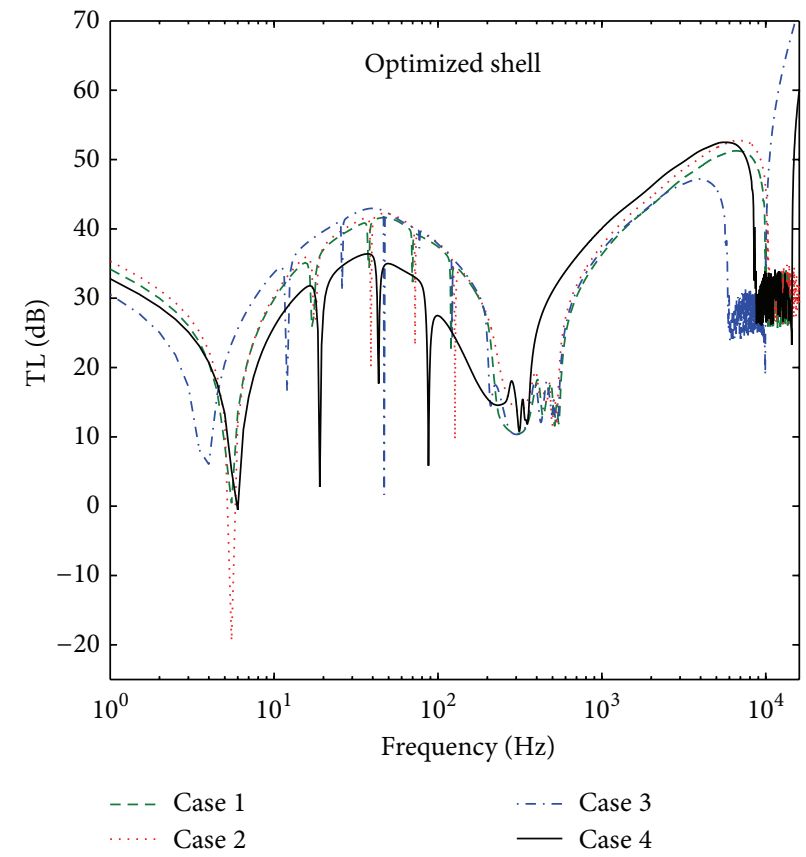

Figure 5: Comparison between TL- $f$ curves for the FGM shells listed in Table 4.

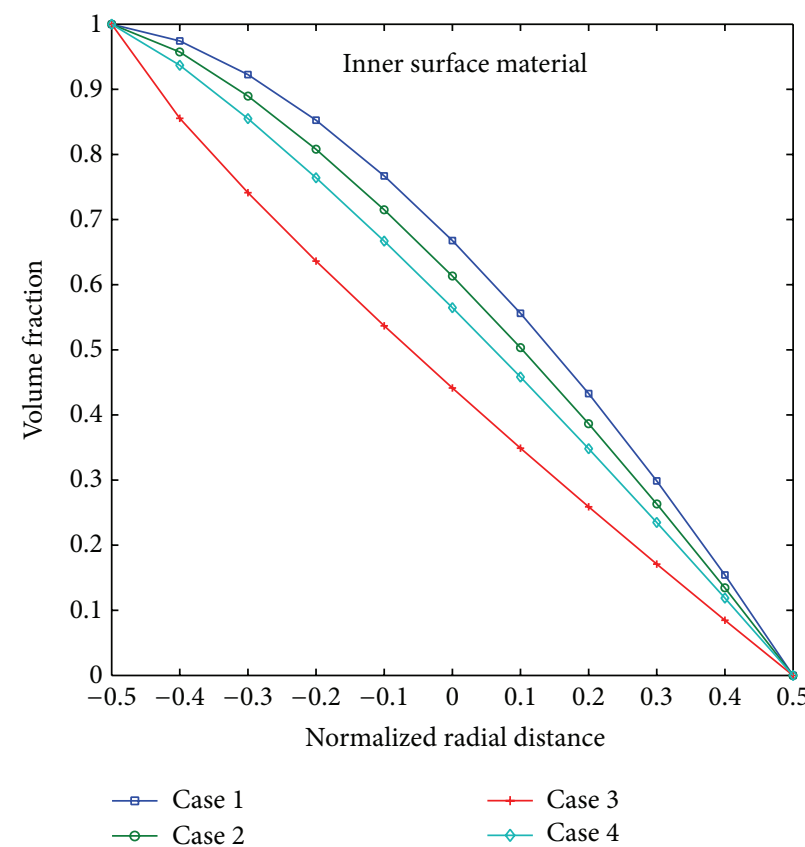

(a)

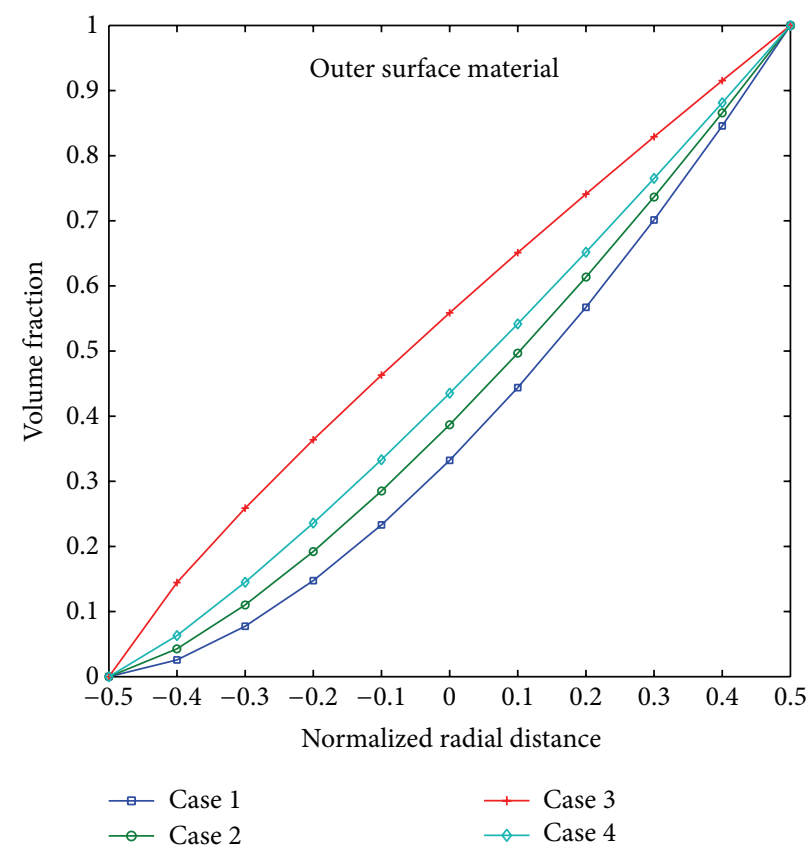

(b)

FIGURE 6: Variation of volume fraction of optimized FGM.

over frequency of $1000 \mathrm{~Hz}$, which have been weighted according to Table 3 . Figure 5 compares the TLs of FGM shells made from different materials with optimized design parameters found by the GA.

Since the aluminum-brass FGM shell was the heaviest, its optimized curve is the highest on the mass control region but its behavior in the stiffness control is the worst. The figure shows that case 1 and case 2 are the most effective in the low and high frequencies range and so they should be used as the first selection material tabulated in Table 4 with optimized value by (26) and (24). Figure 6 shows the variations of the volume fractions in the thickness direction $z$ for all cases of FG cylindrical shell.

In Figure 6, the material properties on the inner surface of the all optimized cylindrical shell are those of aluminum and on the outer surface are those of as in Table 3. As $z$ 
increases $z=-0.5$, the amount of aluminum constituent for the optimized case 1 and case 3 is the highest and the lowest compared to other optimized cases, respectively. This means that the weight constraint of optimized shell at case 1 is better than other cases, while maximum STL is achieved.

\section{Concluding Remarks}

The optimization study of sound transmission across a FGM cylindrical shell has been presented in this paper. The main objective of the optimization is to maximize the sound transmission loss across the structure subjected to weight and the thickness constraint. In order to prevent the softening effect occurrence due to optimization, an additional term is added to the objective function based on the first resonant frequency. Several traditional materials have been chosen for investigation. The power-law exponent, shell radius, thickness of shell, and first resonant frequency have been selected as design variables. Optimization of transmission loss is performed over the frequency range $1000-4000 \mathrm{~Hz}$, where the ear is the most sensitive. The results show that the optimized shell made of aluminum-nickel and aluminum-steel is more effective on the STL in a wide frequency range, while aluminum-brass and aluminum-zircon are only effective in mass control region and stiffness control region, respectively. Based on the result of optimized shell geometries and its volume fraction, minimum weight design with best STL is found for aluminum-nickel and aluminum-steel functionally graded materials.

\section{Conflict of Interests}

The authors declare that there is no conflict of interests regarding the publication of this paper.

\section{References}

[1] M. Yamanouchi, M. Koizumi, T. Hirai, and I. Shiota, "On the design of functionally gradient materials," in Proceedings of the 1st International Symposium on Functionally Gradient Materials, pp. 5-10, Sendai, Japan, 1990.

[2] M. Koizumi, "Functionally gradient materials the concept of FGM," Ceramic Transactions, vol. 34, pp. 3-10, 1993.

[3] H. Yamaoka, M. Yuki, K. Tahara, T. Irisawa, R. Watanabe, and A. Kawasaki, "Fabrication of functionally gradient material by slurry stacking and sintering process," Ceramic Transactions, Functionally Gradient Materials, vol. 34, pp. 165-172, 1993.

[4] J. C. Zhu, Z. D. Yin, and Z. H. Lai, "Fabrication and microstructure of $\mathrm{ZrO}_{2}$-Ni functional gradient material by powder metallurgy," Journal of Materials Science, vol. 31, no. 21, pp. 5829-5834, 1996.

[5] R. Ansari, M. Darvizeh, and M. Hemmatnezhad, "Vibration analysis of FGM cylindrical shells under various boundary conditions," Journal of Agricultural Science and Technology, vol. 5, no. 3, pp. 129-138, 2008.

[6] S. R. Li, X. H. Fub, and R. C. Batrac, "Free vibration of threelayer circular cylindrical shells with functionally graded middle layer," Mechanics Research Communications, vol. 37, pp. 577$580,2010$.
[7] R. L. Williamson, B. H. Rabin, and G. E. Byerly, "FEM study of the effects of inter layers and creep in reducing residual stresses and strains in ceramic-metal joints," Composites Engineering, vol. 5, pp. 851-863, 1995.

[8] J. S. Moya, "Layered ceramics," Advanced Materials, vol. 7, no. 2, pp. 185-189, 1995.

[9] M. M. Gasik and K. R. Lilius, "Evaluation of properties of W$\mathrm{Cu}$ functional gradient materials by micromechanical model," Computational Materials Science, vol. 3, pp. 41-49, 1994.

[10] M. Sasaki and T. Hirai, "Thermal fatigue resistance of CVD $\mathrm{SiC} / \mathrm{C}$ functionally gradient material," Journal of the European Ceramic Society, vol. 14, pp. 257-260, 1994.

[11] R. C. Wetherhold, S. Seelman, and J. Z. Wang, "The use of functionally graded materials to eliminate or control thermal deformation," Composites Science and Technology, vol. 56, no. 9, pp. 1099-1104, 1996.

[12] S. Takezono, K. Tao, E. Inamura, and M. Inoue, “Thermal stress and deformation in functionally graded material shells of revolution under thermal loading due to fluid," JSME International Journal Series A: Mechanics and Material Engineering, vol. 39, pp. 573-581, 1996.

[13] A. Makino, N. Araki, H. Kitajima, and K. Ohashi, "Transient temperature response of functionally gradient material subjected to partial, step wise heating," Transactions of the Japan Society of Mechanical Engineers B, vol. 60, pp. 4200-4206, 1994.

[14] X. D. Zhang, D. Q. Liu, and C. C. Ge, “Thermal stress analysis of axial symmetry functionally gradient materials under steady temperature field," Journal of Functional Materials, vol. 25, pp. 452-455, 1994.

[15] Y. Obata and N. Noda, "Steady thermal stresses in a hollow circular cylinder and a hollow sphere of afunctionally gradient material," Journal of Thermal Stresses, vol. 17, no. 3, pp. 471-487, 1994.

[16] C. T. Loy, K. Y. Lam, and J. N. Reddy, "Vibration of functionally graded cylindrical shells," International Journal of Mechanical Sciences, vol. 41, no. 3, pp. 309-324, 1999.

[17] S. C. Pradhan, C. T. Loy, K. Y. Lam, and J. N. Reddy, "Vibration characteristics of functionally graded cylindrical shells under various boundary conditions," Applied Acoustics, vol. 61, pp. 111$129,2000$.

[18] Y. Y. Tang, J. H. Robinson, and R. J. Silcox, "Sound transmission through a cylindrical sandwich shell with honeycomb core," in Proceedings of the 34 th AIAA Aerospace Science Meeting and Exhibit, 1996.

[19] K. Daneshjou, M. M. Shokrieh, M. G. Moghaddam, and R. Talebitooti, "Analytical model of sound transmission through relatively thick FGM cylindrical shells considering third order shear deformation theory," Composite Structures, vol. 93, no. 1, pp. 67-78, 2010.

[20] S. E. Makris, C. L. Dym, and J. M. Smith, "Transmission loss optimization in acoustic sandwich panels," Journal of the Acoustical Society of America, vol. 79, no. 6, pp. 1833-1843, 1986.

[21] T. Wang, S. Li, and S. R. Nutt, "Optimal design of acoustical sandwich panels with a genetic algorithm," Journal of Applied Acoustics, vol. 70, no. 3, pp. 416-425, 2009.

[22] L. R. Koval, "effects of cavity resonances on sound transmission into a thin cylindrical shell," Journal of Sound and Vibration, vol. 59, no. 1, pp. 23-33, 1978.

[23] M. S. Qatu, Vibration of laminated shells and plates, Elsevier Academic Press, New York, NY, USA, 2004. 
[24] J. N. Reddy, Mechanics of Laminated Composite Plates and Shells, Theory and Analysis, CRC Press, 2nd edition, 2004.

[25] J. Kim and H. Lee J, "Study on sound transmission characteristics of a cylindrical shell using analytical and experimental models," Applied Acoustics, vol. 64, pp. 611-632, 2003.

[26] L. R. Koval, "On sound transmission into a thin cylindrical shell under flight conditions," Journal of Sound and Vibration, vol. 48, pp. 265-275, 1976.

[27] M. J. Crocker, Handbook of Acoustics, John Wiley and Sons, New York, NY, USA, 1998.

[28] C. L. Dym and M. A. Lang, "Transmission of sound through sandwich panels," Journal of the Acoustical Society of America, vol. 56, pp. 1523-1532, 1974.

[29] American Society of Testing and Materials, "Standard practice for determining single-number rating of airborne sound isolation in multi unit building specifications," in Annual Book of ASTM Standards, vol. 04.06, pp. E597-E581, American Society of Testing and Materials, Philadelphia, Pa, USA, 1984. 

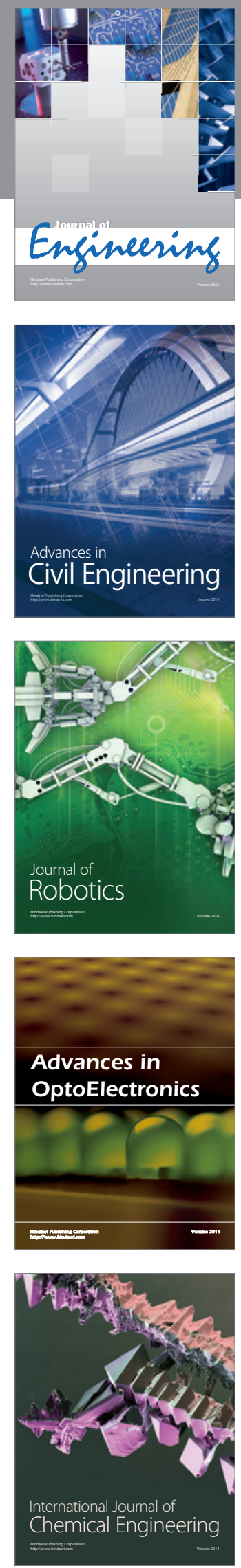

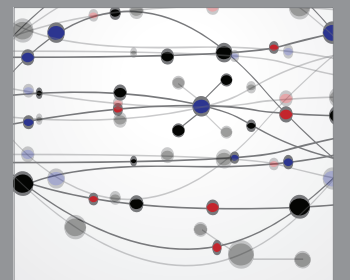

The Scientific World Journal
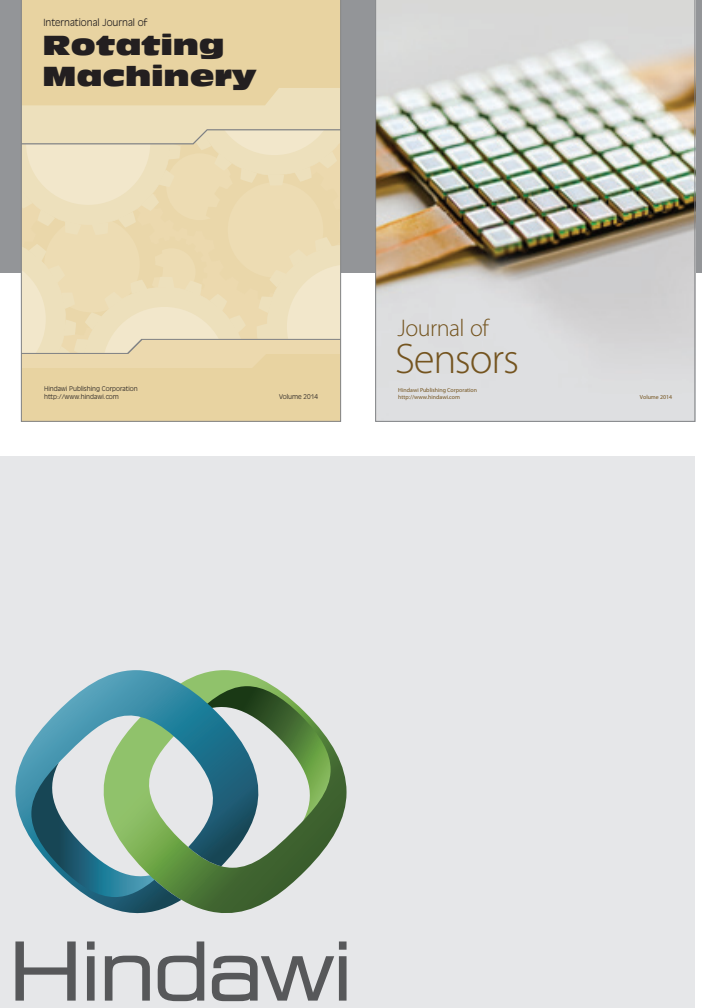

Submit your manuscripts at http://www.hindawi.com
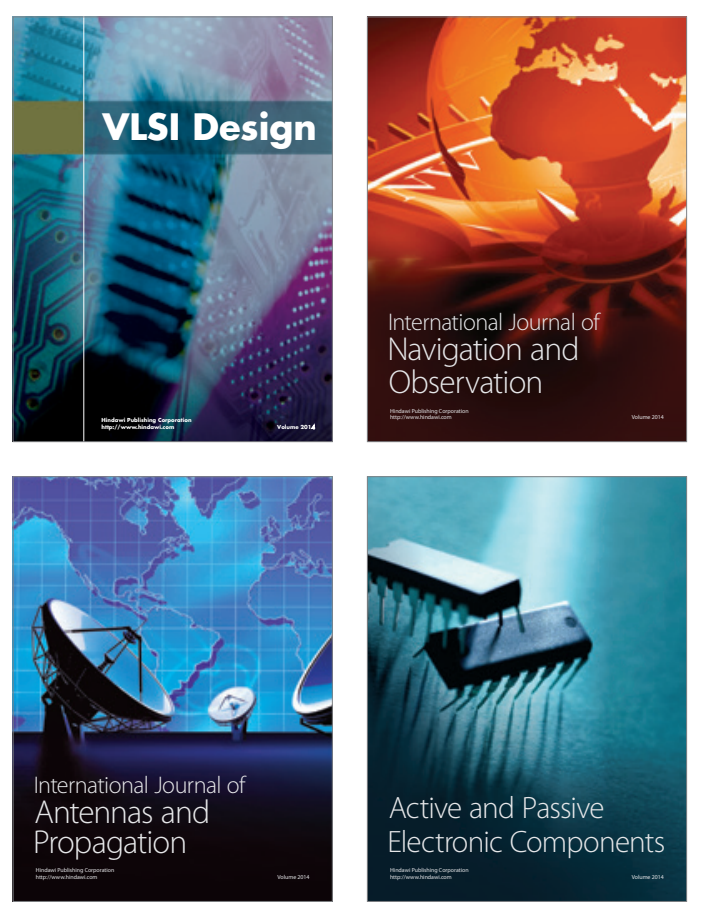
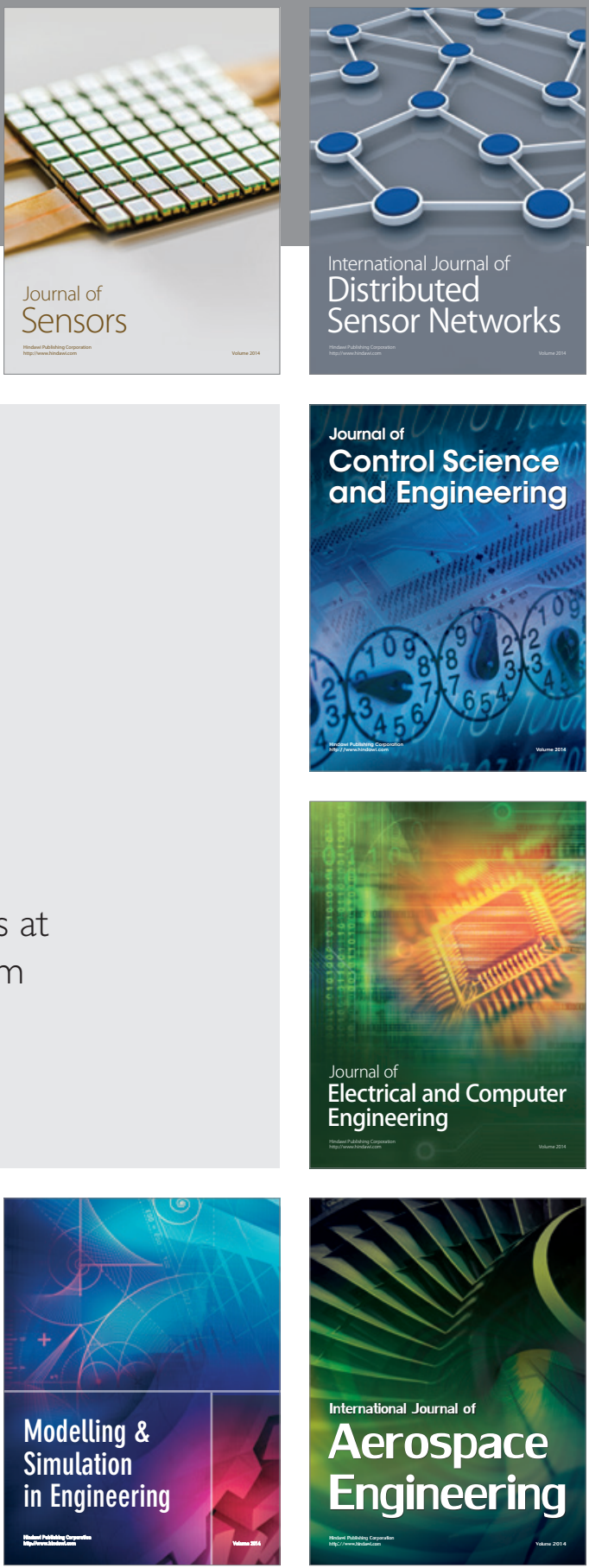

Journal of

Control Science

and Engineering
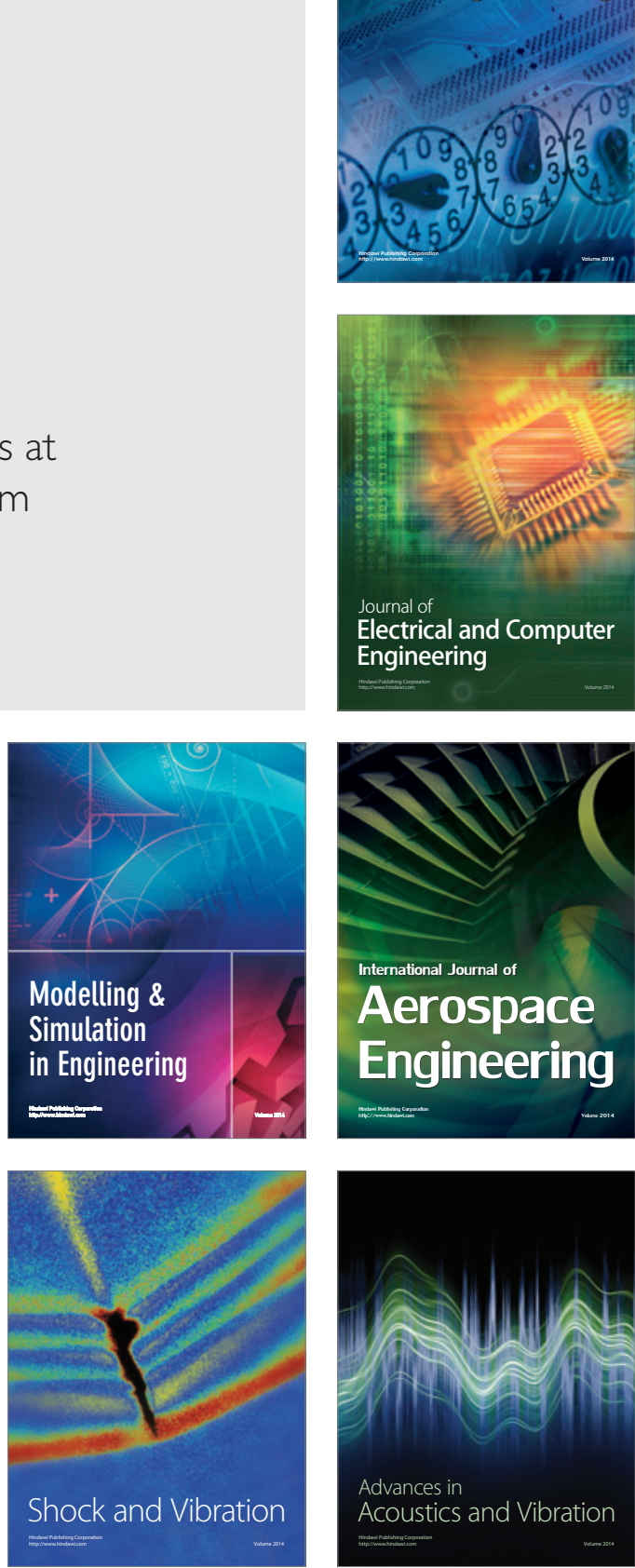